\title{
STRIPPING CROSS SECTIONS IN COLLISIONS OF LIGHT NUCLEI
}

\author{
Yu.M.Shabelski \\ Petersburg Nuclear Physics Institute, \\ Gatchina, St.Petersburg 188350 Russia \\ and \\ D.Treleani \\ Dipartimento di Fisica Teorica dell'Università and INFN, \\ Trieste, I 34014 Italy
}

\begin{abstract}
By evaluating all the contributions of the intermediate states of the multiple scattering theory diagrams, we compute the integrated stripping cross sections of collisions among light nuclei. The resulting expressions have the simple form of a combination of total inelastic cross sections of nuclear reactions with projectile nuclei differing in the atomic mass number. We also check the accuracy of some widely used relations in heavy ion collisions.
\end{abstract}

E-mail shabel@vxdesy.desy.de

E-mail daniel@trieste.infn.it

PACS: 25.60.Dz; 25.75.-q; 25.70.Mn 


\section{Introduction}

Some exclusive cross sections can be expressed as differences of two (or more) total inelastic cross sections. In ref. [1] the probability for one constituent quark of a proton to interact in a collision with nucleus $\mathrm{A}$, while the other two constituent quarks are spectators, was written, in a simplest probabilistic approach, as

$$
V_{1}^{p}(A)=\frac{3}{\sigma_{\text {prod }}^{p A}} \int d^{2} b e^{-2 \sigma_{q} T(b)}\left[1-e^{-\sigma_{q} T(b)}\right],
$$

where $\sigma_{q} \approx \frac{1}{3} \sigma_{\text {inel }}^{p p} \approx 10 \mathrm{mb}$,

$$
T(b)=A \int_{-\infty}^{+\infty} d z \rho(b, z)
$$

and $\rho\left(r=\sqrt{b^{2}+z^{2}}\right)$ is nuclear density.

Eq. (1) can be obviously rewritten as

$$
V_{1}^{p}(A)=\frac{3}{\sigma_{\text {prod }}^{\text {pA }}}\left[\int d^{2} b\left(1-e^{-3 \sigma_{q} T(b)}\right)-\int d^{2} b\left(1-e^{-2 \sigma_{q} T(b)}\right)\right],
$$

where the two integrals represent the absorption cross sections of a threequark and of a two-quark system respectively.

In the present note we show that in the case of collisions among two light nuclei similar expressions hold to a very high accuracy, accounting in fact for the contributions of interference terms, loop diagrams, etc. The processes which we consider are nuclear reactions with stripping of one or few nucleons and we compute the corresponding integrated cross sections and the average number of interacting nucleons. The main tools of our analysis are the multiple scattering theory (Glauber theory) and the AGK cutting rules [2]. We study first the case of tritium-deuteron $(t d)$ interaction and we discuss afterwards how the results are generalized to the case of heavier nuclei.

The region of validity of the present analysis is the energy region where the multiple scattering theory is available, namely from a few hundred $\mathrm{MeV}$ per nucleon to superhigh energies, where inelastic screening corrections have to be taken into account. For simplicity we neglect the difference between $p p$ and $p n$ interactions. 


\section{Multiple scattering theory diagrams for the total cross section}

The simplest nuclear target is deuteron. One can therefore start considering the elastic scattering amplitude, or the total cross section, for $p d, d d$ and $t d$ interactions. The multiple scattering theory diagrams for the processes are shown in Figs. 1, 2 and 3, where the interacting nucleons are represented by dots and the interactions by solid lines. Non-interacting nucleons are not shown. The number at a side of each diagram is the corresponding combinatorial factor $l_{i}$ and $\Delta_{n, i}$ is the contribution of the diagram to the total cross section. The index $n$ in $\Delta_{n, i}$ represents the number of interactions and the index $i$ allows one to distinguish between different diagrams at the same order in the number of interactions. As an example, in Fig. 3 the combinatorial factor $l_{i}$ is equal to 6 for the diagram corresponding to $\Delta_{2, b}$, since there are three possibilities to choose the non-interacting nucleon in tritium and two possibilities to choose the interacting nucleon in deuteron.

The total cross section of two interacting nuclei is represented by the sum of all possible diagrams in the multiple scattering theory, which, by introducing the quantities

$$
\Delta_{n}=\sum_{i} l_{i} \Delta_{n, i}
$$

is expressed as:

$$
\sigma_{\text {tot }}=\sum_{n}^{A \cdot B}(-1)^{n-1} \Delta_{n}
$$

\section{Cross section for stripping one nucleon in $t d$ collisions}

The simplest stripping process to consider is the one where one nucleon, of a fast $t$-nucleus, is scattered with a large transverse momentum with respect to its Fermi momentum, while the two other nucleons are spectators. From the point of view of the unitarity relation the needed intermediate states can be found when the nucleon-nucleon interaction blob, Fig. 4a, is cut. In the case of the cut between two interactions, as in Fig. 4b, the probability to find a nucleon with comparatively large transverse momentum is suppressed by the nuclear form factor.

The cross section corresponding to a given cut diagram is deternimed by the AGK cutting rules [2, 3, 4] which were first obtained for reggeon

diagrams [2] and later were proved for hadron-nucleus [3] and nucleus-nucleus 
[4] interactions. As an example, the cross section of the process corresponding to a cut diagram of the kind shown in Fig. 4a is equal to the contribution to the total cross section of the same uncut diagram $\left(\Delta_{2 c}\right.$ in the considered case) multiplied by the AGK [2, 3, 4] factor

$$
D_{n}^{(k)}=(-1)^{n+k} 2^{n-1} \frac{n !}{(n-k) ! k !},
$$

where $n$ is the number of interactions and $k$ the number of cut blobs. In Fig. 4a $n=2$ and $k=1$.

The one-cut-blob contribution to the cross section for stripping one-nucleon in tritium-deuteron interactions, in accordance with the diagrams in Fig. 3 and with Eq. (6), is expressed as

$$
\begin{aligned}
\sigma_{1}^{(1)} & =6 \Delta_{1}-4\left(3 \Delta_{2, a}+6 \Delta_{2, b}+6 \Delta_{2, c}\right)+12\left(12 \Delta_{3, a}+2 \Delta_{3, b}+6 \Delta_{3, c}\right)-(7) \\
& -32\left(3 \Delta_{4, a}+6 \Delta_{4, b}+6 \Delta_{4, c}\right)+480 \Delta_{5}-192 \Delta_{6} .
\end{aligned}
$$

A different way to produce a stripped nucleon is to cut two blobs as in Fig. 3 (2a). A further possibility is to cut two blobs in Fig. 3 (3a). In this last case one needs however to include an additional factor $1 / 3$ since only one combination out of the possible three gives the needed state. After accounting for all factors, the contribution of the two-cut-blob terms to the one-nucleon stripping cross section in tritium-deuteron reactions is written as

$$
\sigma_{1}^{(2)}=6 \Delta_{2, a}-48 \Delta_{3, a}+48\left(\Delta_{4, a}+\Delta_{4, b}+\Delta_{4, c}\right)-192 \Delta_{5}+96 \Delta_{6} .
$$

All other possible cuts of the multiple scattering theory diagrams give more than one stripped nucleon. The total cross section for one-nucleonstripping reactions, $\sigma_{1}$, is therefore equal to the sum $\sigma_{1}^{(1)}+\sigma_{1}^{(2)}$ which can be written as

$$
\begin{aligned}
\sigma_{1} & =3\left[2 \Delta_{1}-\left(2 \Delta_{2, a}+8 \Delta_{2, b}+8 \Delta_{2, c}\right)+\left(32 \Delta_{3, a}+8 \Delta_{3, b}+24 \Delta_{3, c}\right)-(9)\right. \\
& \left.-8\left(2 \Delta_{4, a}+6 \Delta_{4, b}+6 \Delta_{4, c}\right)+96 \Delta_{5}-32 \Delta_{6}\right] .
\end{aligned}
$$

The total inelastic $t d$ and $d d$ cross sections can be expressed in the form [四] (also see below) :

$$
\sigma_{\text {inel }}=\sum_{n}^{A \cdot B}(-1)^{n-1} 2^{n-1} \Delta_{n}
$$

The values of $\Delta_{n}$ are different in $t d$ and in $d d$ interactions. One may however notice that the value of each $\Delta_{n, i}$, in $t d$ interactions, is determined by the nucleon-nucleon cross section parameters and by the radii of tritium and 
deuteron, which are present in the result with coefficients depending on the kind of diagram considered. If one forces one of the terms $\Delta_{n, i}$ to be the same in $t d$ and in $d d$ interactions, by rescaling for example the deuteron radius, all other terms $\Delta_{n, i}$, when rescaled in the same way, are therefore also forced to coincide. One can then compute the $d d$ process in multiple scattering theory by changing the radius of the projectile deuteron in such a way as to obtain equal values for all $\Delta_{n, i}$ in $t d$ and in $d d$ reactions. Obviously one cannot compare the value of $\sigma_{\text {inel }}^{d d}$ which is obtained in this way with the experimental value. The expression of $\sigma_{\text {inel }}^{d d}$, that one obtains with this procedure, allows however one to write in a rather compact form the stripping cross section in terms of the difference $\sigma_{\text {inel }}^{t d}-\sigma_{\text {inel }}^{d d}$. As a consequence of the cancellation between many of the terms appearing in $\sigma_{\text {inel }}^{t d}$ and in $\sigma_{\text {inel }}^{d d}$ one can in fact write

$$
\begin{aligned}
\sigma_{\text {inel }}^{t d}-\sigma_{\text {inel }}^{d d} & =2 \Delta_{1}-\left(2 \Delta_{2, a}+8 \Delta_{2, b}+8 \Delta_{2, c}\right)+\left(32 \Delta_{3, a}+8 \Delta_{3, b}+(11\right. \\
& \left.+24 \Delta_{3, c}\right)-8\left(2 \Delta_{4, a}+6 \Delta_{4, b}+6 \Delta_{4, c}\right)+96 \Delta_{5}-32 \Delta_{6}
\end{aligned}
$$

which is precisely one third of $\sigma_{1}$ as expressed in Eq.(9):

$$
\sigma_{1}=3\left(\sigma_{\text {inel }}^{t d}-\sigma_{\text {inel }}^{d d}\right)
$$

\section{Cross section for stripping two nucleons in $t d$ collisions}

The cross section $\sigma_{2}$, for stripping two nucleons of tritium, in $t d$ interaction, can be computed analogously to the computation of $\sigma_{1}$ in the previous paragraph. In the process the $t$-nucleus dissociates into one spectator nucleon and two nucleons with transverse momentum large as compared with their Fermi momentum. The cuts to be taken into account in the multiple scattering theory diagrams are those involving two tritium nucleons. One obtains:

$$
\begin{aligned}
\sigma_{2}^{(2)} & =2\left(6 \Delta_{2, b}+6 \Delta_{2, c}\right)-12\left(8 \Delta_{3, a}+2 \Delta_{3, b}+6 \Delta_{3, c}\right)+ \\
& +48\left(2 \Delta_{4, a}+5 \Delta_{4, b}+5 \Delta_{4, c}\right)-768 \Delta_{5}+384 \Delta_{6} .
\end{aligned}
$$

Analogously to the case discussed in the previous Sect., one has to keep into account also of the contribution where the two stripped nucleons are originated in processes where three or more interaction blobs are cut. The simplest example is the diagram in Fig. 3 (3a). The contribution to $\sigma_{2}$ from three cut blobs is equal to

$$
\sigma_{2}^{(3)}=48 \Delta_{3, a}-32\left(3 \Delta_{4, a}+3 \Delta_{4, b}+3 \Delta_{4, c}\right)+576 \Delta_{5}-384 \Delta_{6} .
$$


In a similar way the contribution from four cut blobs is :

$$
\sigma_{2}^{(4)}=24 \Delta_{4, a}-96 \Delta_{5}+96 \Delta_{6}
$$

The expression of the sum of all contributions, where two tritium nucleons are stripped in $t d$ interaction, $\sigma_{2}=\sum \sigma_{2}^{(i)}$, is therefore

$$
\begin{aligned}
\sigma_{2} & =3\left[\left(4 \Delta_{2, b}+4 \Delta_{2, c}\right)-\left(16 \Delta_{3, a}+8 \Delta_{3, b}+24 \Delta_{3, c}\right)+\right. \\
& \left.+\left(8 \Delta_{4, a}+48 \Delta_{4, b}+48 \Delta_{4, c}\right)-96 \Delta_{5}+32 \Delta_{6}\right] .
\end{aligned}
$$

As for $\sigma_{1}$ in the previous paragraph, by making use of Eq. (10) one obtains

$$
\sigma_{2}=3\left(2 \sigma_{\text {inel }}^{d d}-\sigma_{\text {inel }}^{t d}-\sigma_{\text {inel }}^{p d}\right) .
$$

\section{$5 \quad$ Cross section for stripping three nucleons in $t d$ collisions}

The cross section of the process where all the three tritium-nucleons are scattered with a transverse momentum larger with respect to their Fermi momentum, $\sigma_{3}$, can be calculated analogously :

$$
\begin{gathered}
\sigma_{3}^{(3)}=4\left(2 \Delta_{3, b}+6 \Delta_{3, c}\right)-32\left(3 \Delta_{4, b}+3 \Delta_{4, c}\right)+384 \Delta_{5}-256 \Delta_{6}, \\
\sigma_{3}^{(4)}=8\left(6 \Delta_{4, b}+6 \Delta_{4, c}\right)-384 \Delta_{5}+384 \Delta_{6}, \\
\sigma_{3}^{(5)}=96 \Delta_{5}-192 \Delta_{6} \\
\sigma_{3}^{(6)}=32 \Delta_{6} .
\end{gathered}
$$

and

$$
\sigma_{3}=\sum \sigma_{2}^{(i)}=\left(8 \Delta_{3, b}+24 \Delta_{3, c}\right)-\left(48 \Delta_{4, b}+48 \Delta_{4, c}\right)+96 \Delta_{5}-32 \Delta_{6}
$$

which can be written in the form

$$
\sigma_{3}=\sigma_{\text {inel }}^{t d}-3 \sigma_{\text {inel }}^{d d}+3 \sigma_{\text {inel }}^{p d} .
$$

\section{Test of a few relations in nucleus-nucleus interactions}

The equations derived in the previous paragraphs are of rather general validity in multiple scattering theory. One has therefore the possibility to test 
a few well known relations which are widely used in nucleus-nucleus interactions.

The total inelastic tritium-deuteron cross section is equal to the sum of $\sigma_{1}, \sigma_{2}$ and $\sigma_{3}$. By using the explicit expressions in Eq.s (9), (16) and (22) one can write:

$$
\begin{aligned}
\sigma_{\text {inel }}^{t d} & =6 \Delta_{1}-\left(6 \Delta_{2, a}+12 \Delta_{2, b}+12 \Delta_{2, c}\right)+\left(48 \Delta_{3, a}+8 \Delta_{3, b}+\right. \\
& \left.+24 \Delta_{3, c}\right)-\left(24 \Delta_{4, a}+48 \Delta_{4, b}+48 \Delta_{4, c}\right)+96 \Delta_{5}-32 \Delta_{6}
\end{aligned}
$$

which, recalling the definition of $\Delta_{n}$ in Eq.(4), shows that Eq. (10) is an exact relation in multiple scattering theory.

A similar conclusion holds for the average number of interacting nucleons of the incident tritium nucleus, $\left\langle N_{A}\right\rangle$. To obtain the average number one in fact writes

$$
\sigma_{\text {inel }}^{t d}<N>=\sigma_{1}+2 \sigma_{2}+3 \sigma_{3}
$$

and, after substituting the explicit expressions of $\sigma_{1}, \sigma_{2}$ and $\sigma_{3}$, one obtains

$$
\sigma_{1}+2 \sigma_{2}+3 \sigma_{3}=6 \Delta_{1}-6 \Delta_{2, a}=3 \sigma_{\text {inel }}^{p d}
$$

in agreement with the general expression of $\left\langle N_{A}\right\rangle$ [5].

\section{More general expressions}

We have performed analogous calculations in the case of interactions among various different pairs of light nuclei $\left(d d, d^{4} H e, t t, t^{4} H e\right)$. In all cases the results are the same, namely they are of the kind of Eqs. (12), (17) and (23). The cross sections for stripping one, two, three, etc. nucleons from nucleus $A$, in collisions among the two nuclei $A$ and $B$, are written as

$$
\begin{gathered}
\sigma_{1}^{A B}=A\left(\sigma_{\text {inel }}^{A, B}-\sigma_{\text {inel }}^{A-1, B}\right) \\
\sigma_{2}^{A B}=\frac{A(A-1)}{2 !}\left(-\sigma_{\text {inel }}^{A, B}+2 \sigma_{\text {inel }}^{A-1, B}-\sigma_{\text {inel }}^{A-2, B}\right) \\
\sigma_{3}^{A B}=\frac{A(A-1)(A-2)}{3 !}\left(\sigma_{\text {inel }}^{A, B}-3 \sigma_{\text {inel }}^{A-1, B}+3 \sigma_{\text {inel }}^{A-2, B}-\sigma_{\text {inel }}^{A-3, B}\right)
\end{gathered}
$$

with an obvious generalization to the case where the number of stripped nucleons is larger than three. As in the case of tritium, previously discussed, in the expressions above the inelastic cross sections have to be computed by taking, for the nuclei with atomic mass number $A-1, A-2$ and $A-3$, the same radius as for the nucleus with atomic mass number $A$. 
We have also checked that the expression giving the average number of interacting nucleons of the projectile nucleus $A$, actually [5]

$$
<N>_{A B}=A \sigma_{\text {inel }}^{N B} / \sigma_{\text {inel }}^{A B},
$$

is exact at all orders in multiple scattering theory.

\section{Conclusions}

By making use of the AGK cutting rules we have computed the stripping cross sections in collisions involving light nuclei. The expressions which we have obtained can be formally written in a compact way as differences between total inelastic cross sections of reactions where the projectile nuclei differ in the atomic mass number. The total inelastic cross sections, which are used to express the stripping cross sections, are not the experimental quantities. They represent formal expressions to be evaluated in multiple scattering theory, by using the same value of nuclear radius for the different projectile nuclei. All stripping cross sections, in interactions among light nuclei, are therefore written in a convenient way as combinations of the same quantities, which are easily computed in the multiple scattering theory formalism. Multiple scattering theory and the AGK cutting rules allow therefore one fix the normalization of the spectra of the stripped nucleons. The normalization in fact is not a minor problem [6], because of the difficulty of constructing explicitly the ortonormal set of scattering states of a process where various different scattering channels and bound states are present. Which is precisely the case of the stripping reactions. One in fact obtains a non-trivial link between the different stripping cross sections, namely the relations (27), (28) and (29).

In the present note we have also tested some widely used relations in nuclear collisions, Eq.s (10) and (29), which have been shown to be accurate at all orders in the multiple scattering theory.

Some numerically small corrections to all our results can appear from the contributions of cuts between nucleon-nucleon interaction blobs, as shown in Fig. 4b, see discussion in Sect. 3. One has also to point out that, as one can see from Eq. (24), the numerically small cross section of "stripping zero nucleon", i.e. of the process $t+d \rightarrow t+p+n$, is not included into $\sigma_{\text {inel }}^{t d}$.

We finally remark that, while the expressions which we have worked out refer to the stripping cross sections, our approach can be easily generizable to reactions of different kind, as for example pion production and Drell-Yan pair production.

\section{Acknowledgements}


This work is supported in part by INTAS grant 93-0079 and by the Italian Ministry of University and of Scientific and Technological Research, by means of the Fondi per la Ricerca scientifica - Università di Trieste.

\section{Figure captions}

Fig. 1. Multiple scattering theory diagrams for $p d$ elastic scattering amplitude. (See text for definitions.)

Fig. 2. Multiple scattering theory diagrams for $d d$ elastic scattering amplitude. (See text for definitions.)

Fig. 3. Multiple scattering theory diagrams for $t d$ elastic scattering amplitude. (See text for definitions.)

Fig. 4. The examples of cut of the diagram Fig. 3 (2c): cut of the blob of nucleon-nucleon interaction (a) and cut between blobs (b).

\section{References}

[1] V.V.Anisovich, Yu.M.Shabelski and V.M.Shekhter. Nucl.Phys. B133 (1978) 477.

[2] V.A.Abramovsky, V.N.Gribov and O.V.Kancheli. Yad.Fiz. 18 (1973) 595 .

[3] Yu.M.Shabelski. Nucl.Phys. B132 (1978) 491.

[4] Yu.M.Shabelski. Yad.Fiz. 47 (1988) 1612.

[5] A.Bialas, M.Bleszynski and W.Czyz. Nucl.Phys. B11 (1976) 461.

[6] L.Bertocchi and D.Treleani. Nuovo Cimento A36 (1976) 1. 


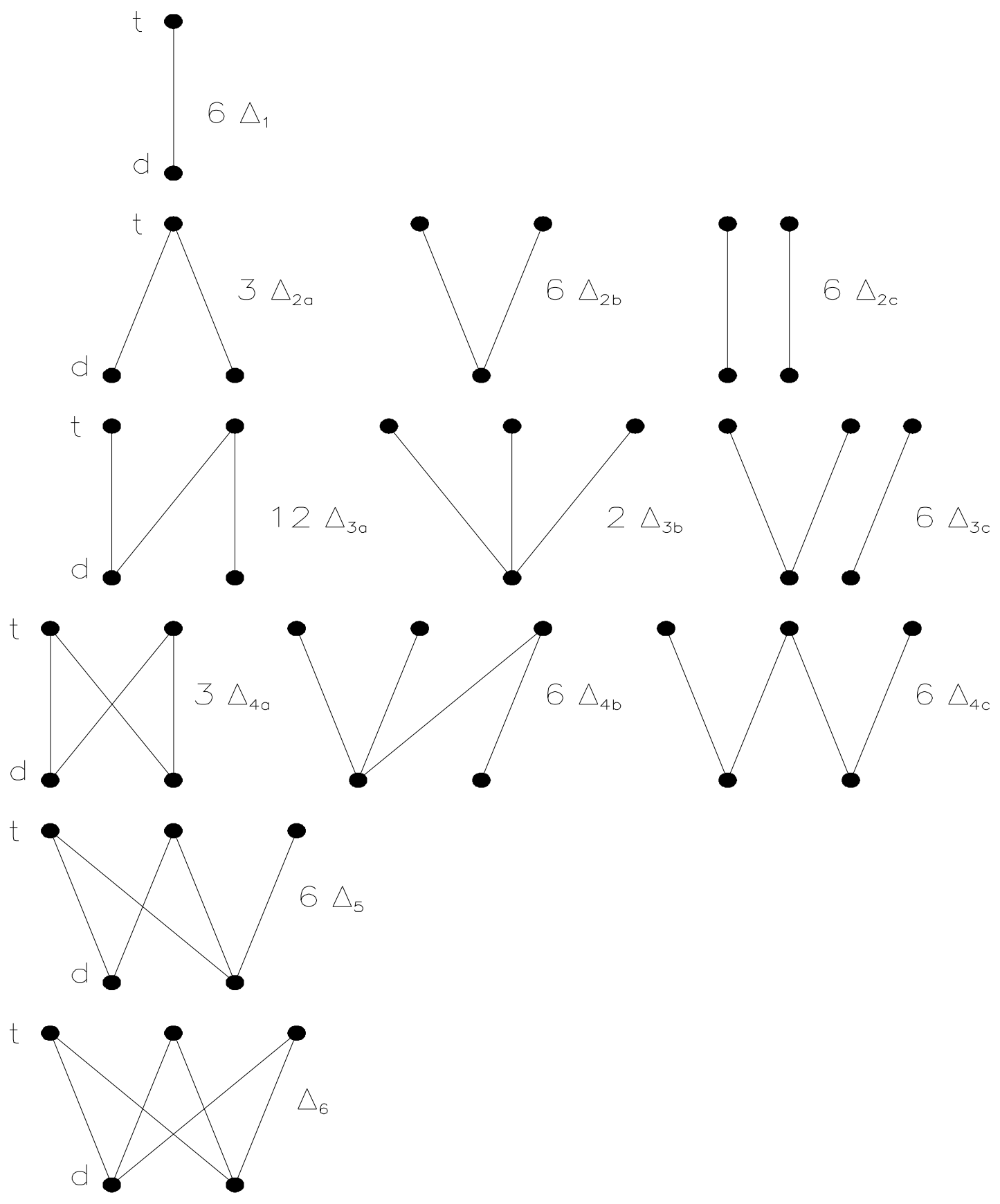

Fig. 3 

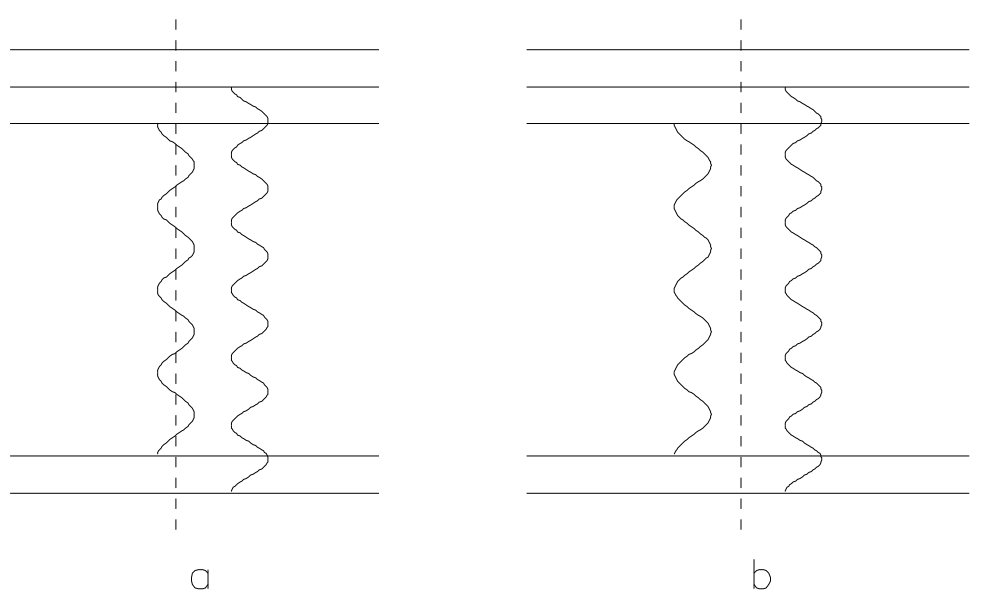

Fig. 4 

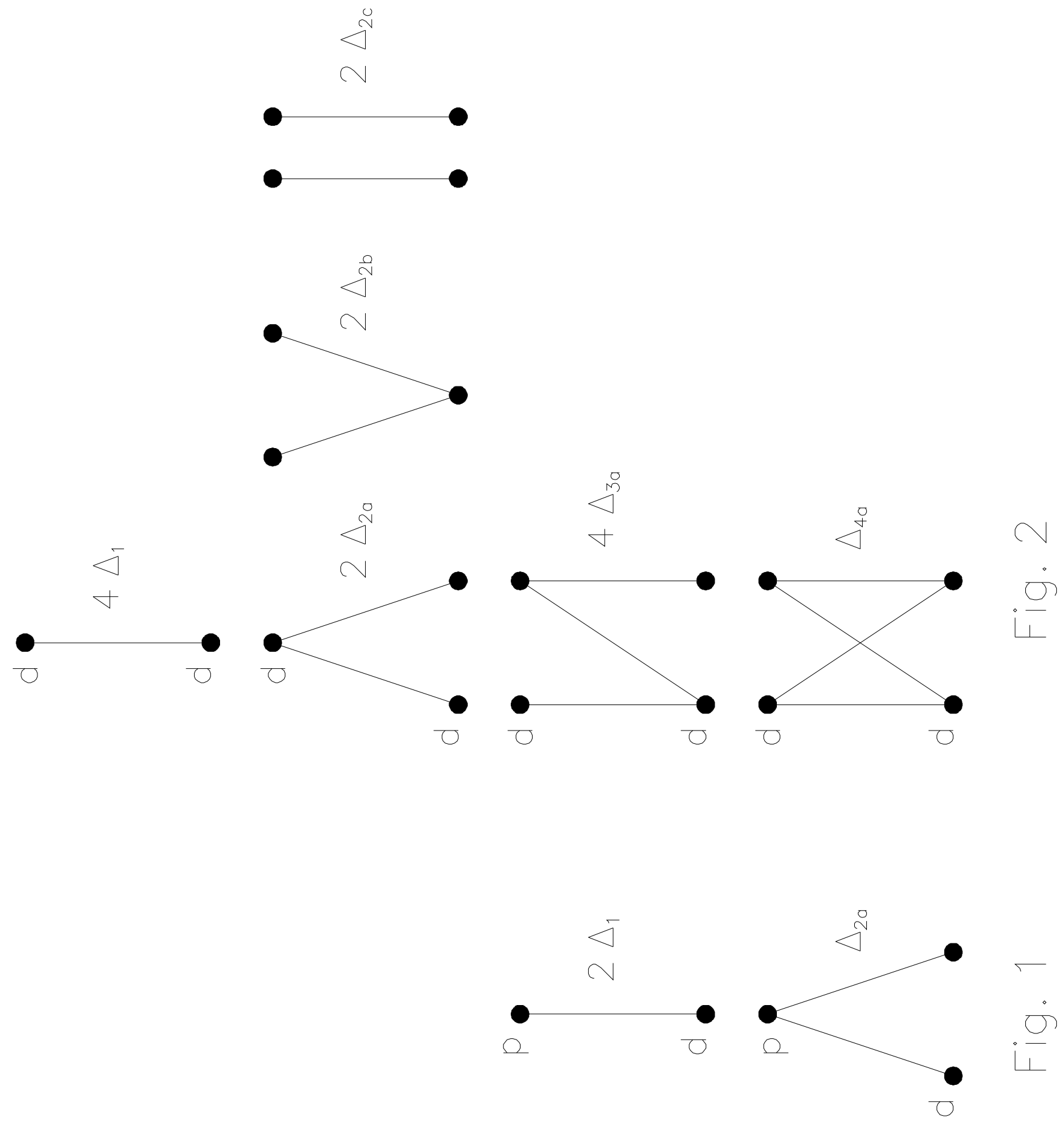